Research Article

\title{
Examining the Accuracy of Network RTK and Long Base RTK Methods with Repetitive Measurements
}

\author{
Tamer Baybura ${ }^{D},{ }^{1}$ İbrahim Tiryakioğlu, ${ }^{1}$ Mehmet Ali Uğur, ${ }^{1}$ Halil İbrahim Solak, ${ }^{2}$ \\ and Şeyma Şafak ${ }^{2}$ \\ ${ }^{1}$ Department of Geomatics Engineering, Afyon Kocatepe University, Afyonkarahisar 03200, Turkey \\ ${ }^{2}$ Distance Education Vocational School, Afyon Kocatepe University, Afyonkarahisar 03200, Turkey \\ Correspondence should be addressed to Tamer Baybura; tbaybura@aku.edu.tr
}

Received 12 July 2019; Revised 3 October 2019; Accepted 29 October 2019; Published 18 November 2019

Academic Editor: Xinyu Liu

Copyright @ 2019 Tamer Baybura et al. This is an open access article distributed under the Creative Commons Attribution License, which permits unrestricted use, distribution, and reproduction in any medium, provided the original work is properly cited.

\begin{abstract}
Real-time kinematic (RTK) technique is important for mapping applications requiring short measure time, the distance between rover and base station, and high accuracy. There are several RTK methods used today such as the traditional RTK, long base RTK (LBRTK), network RTK (NRTK), and precise point positioning RTK (PPP-RTK). NRTK and LBRTK are popular with the advantage of the distance, the time, and accuracy. In the present study, the NRTK and LBRTK measurements were compared in terms of accuracy and distance in a test network with 6 sites that was established between 5 and $60 \mathrm{~km}$. Repetitive NRTK and LBRTK measurements were performed on 6 different days in 2015-2017-2018 and additionally 4 campaigns of repetitive static measurements were carried out in this test network. The results of NRTK and LBRTK methods were examined and compared with all relevant aspects by considering the results of the static measurements as real coordinates. The study results showed that the LBRTK and NRTK methods yielded similar results at base lengths up to $40 \mathrm{~km}$ with the differences less than $3 \mathrm{~cm}$ horizontally and $4 \mathrm{~cm}$ vertically.
\end{abstract}

\section{Introduction}

Nowadays, it is possible to get accurate and precision coordinates anywhere in seconds thanks to GNSS technology $[1,2]$. The RTK method, using the differential GNSS technique, is based on network principle and serving quick and practical measurements. Some methods to get accuracy and precision are long base real-time kinematic (LBRTK), network realtime kinematic (NRTK), and precise point positioning real-time kinematic (PPP-RTK). With real-time satellite orbit and clock corrections, several PPP services have been developed so far and PPP-RTK is one of the services. In PPP-RTK method, data of the single GNSS receiver are evaluated with different corrections like precise satellite orbits and clocks, ionospheric delays, and satellite phase biases to compute receiver's position with $\mathrm{cm}$ accuracy. Solution of ambiguity resolution quickly and effectively has a big importance for the PPP-RTK technique and several methods have been developed for the solution. Long solution time of ambi- guity resolution is a problem for the use of PPP-RTK technique in instant applications requiring high accuracy [3-5].

On the other hand, with long base real-time kinematic (LBRTK) and network real-time kinematic (NRTK), which is the main subject of this study, the base length of $3-5 \mathrm{~km}$ could be expanded to $100 \mathrm{~km}$ with short measurement time [6-8]. Real-time LBRTK transmission is made with a rover receiver, and all corrections are computed from one station. NRTK communicates real-time with the control centre of a fixed station network and corrects real-time transmissions with a rover receiver, which is done with computing solutions, such as the VRS (virtual reference stations), MAC (Master Auxiliary Concept), PRS (pseudo reference stations), and FKP (Flächenkorrekturparameter) [9].

For short distances $(\sim 10 \mathrm{~km}$, depending on the ionospheric conditions), up to 10 kilometres, a common practice is to neglect the ionospheric effect. For this reason, the reduction in differential ionospheric effects is one of the most important stages in ambiguity resolution (AR) improvement 
and, therefore, for accurate medium and long base kinematic positioning $[10,11]$. On GNSS networks, most of the modelling algorithms in distance-dependent ionospheric errors are accepted as these errors might be interpolated linearly; in other words, their spatial wavelengths are larger than the network station separation [12].

$\mathrm{AR}$ is another important criterion in network RTK solution time. For longer distances, differential ionospheric residuals become larger and might hamper the AR process (or, sometimes, make it impossible).

The objective of this study is comparing the coordinates obtained from LBRTK, NRTK, and long-term static GNSS measurements. The LBRTK measurements were made depending on the AFKU station that was established at Afyon Kocatepe University. The NRTK measurements were made with respect to the CORS-TR network.

\section{Materials and Methods}

2.1. Real-Time Kinematic. The kinematic measurement method is divided into two groups: the traditional RTK (real-time kinematic) and the NRTK (network RTK). The traditional RTK consists of two components, i.e., the base and rover stations. The corrections that are obtained from the base station are sent to the rover stations with the help of radio modems. The biggest problem of this system is that the RTK corrections limit the transfer distances within 5$10 \mathrm{~km}$ because of the limited power of their radio modems. For the purpose of eliminating the deficiencies of this system, fixed GNSS stations that provide continuous data transfer through GSM modems instead of radio modems are being established. These networks are called CORS (Continuously Operating Reference Station) or network RTK. Although CORS stations are being used for the purpose of NRTK, it is possible to use them for LBRTK. The basic purpose of establishing NRTK GNSS networks (CORS) is to eliminate the errors that differ depending on the distance (ionosphere, troposphere, orbit, etc.) and to send the corrections to long distances in real time. This system consists of 3 or more stable stations that make continuous observations $[13,14]$. NRTK necessitates an ambiguity resolution "engine" to fix integer ambiguities in static multiple reference receivers in stations with known positions to form the network [9]. The reference station separation is generally limited to less than $100 \mathrm{~km}$ for fast and accurate ambiguity resolution. When the station count increases, corrections are attained [15]. Many correction computing solutions are currently available, such as the VRS (virtual reference stations), MAC (Master Auxiliary Concept), PRS (pseudo reference stations), and FKP (Flächenkorrekturparameter) methods [14, 16, 17]. The VRS technique is currently the most popular and efficient method of transmitting corrections through a data link to the network users for RTK positioning.

The biggest disadvantage of network RTK is the installation cost of the system with its components because there is a need for 3 or more fixed stations in the system. The disadvantages of these two systems have caused new approaches to emerge. Long base RTK is one of these approaches. GSM modems are used in long base RTK instead of radio modems used in the traditional RTK Method. Thanks to GSM modems, the distance base and rover stations reached approximately 100 kilometres. Since the CORS system in Turkey requires fees for usage, LBRTK can be considered an economical and useful method. There are 100 LBRTK stations in Turkey and they are used for mapping activities and deformation monitoring for free.

The comparisons of traditional, long base, and network RTK are given in Table 1.

2.2. RTK Errors. GNSS positioning accuracy is dependent on 5 factors:

(i) Ionospheric errors

(ii) Tropospheric errors

(iii) Signal obstructions and multipath

(iv) Geometric configuration of satellites

(v) Other errors

2.2.1. Ionospheric Errors. GNSS signal propagation depends on the state of the ionosphere as intensive irregularities and/or gradients of the electron density change the propagating wave parameters [11]. Ionospheric scintillation has a great impact on radio propagation and electronic system performance; thus, it is extensively studied currently. The influence of scintillation on global navigation satellite system (GNSS) is particularly evident, making GNSS an effective medium to study characteristics of scintillation. Ionospheric scintillation varies greatly in relation with temporal and spatial distribution [19].

Severe solar radio bursts (a type of ionospheric storm) might disrupt GNSS communication at a significant level because they are the source of radio noise. Ionospheric storms are characterized by some "indices" that are measured and published daily as Kp [20].

The Kp index is determined by calculating the changes stemming from solar activities in the magnetic field of the earth and ionospheric storms with 13 magnetism stations. If the $\mathrm{Kp}$ index of an area is small, it is understood that the magnetic state is stable. These index values are classified as 0-9; if the value is below 2, magnetism is defined as very stable; if the value is 3 , magnetism is defined as unstable; and if the value is 4 and above, magnetism is defined as active or unstable. The disruption between the Kp index and GNSS signals is proportional. However, with various ionospheric models, these disruptions might be eliminated. The stations that were included in the present study were located in the middle latitudes (Afyon, Turkey), and it was stated in various studies that the Kp index is lower in the middle latitudes $[11,20,21]$.

2.2.2. Tropospheric Errors. GNSS signals are affected while passing throughout the troposphere. The troposphere causes a delay in the GNSS signals with respect to the different heights above sea level. Each of $1 \mathrm{~cm}$ error while defining the tropospheric delay causes $3 \mathrm{~cm}$ error at the up coordinate of the site. Most of the GNSS receivers have a tropospheric 
TABLE 1: Comparison of 3 RTK methods [18].

\begin{tabular}{lccc}
\hline & Traditional RTK & Long base RTK & Network RTK \\
\hline Reduces startup costs to purchase an RTK system & Low & Low & High \\
Quickly setup and start surveying & Slow & Fast & Fast \\
License fee for UHF radios & Yes & No & No \\
Fee for communications & No & Yes & Yes \\
Distance to base & $5-10 \mathrm{~km}$ & $50-100 \mathrm{~km}$ & $50-100 \mathrm{~km}$ \\
Distance-dependent errors & High & Investigated in this study & Low \\
\hline
\end{tabular}

model which reduces the tropospheric impacts. In RTK measurements, if possible, the base and rover stations are selected at approximately the same heights in terms of reducing the tropospheric impacts. In the literature, it has been stated that the effect of the height difference is limited as below 300 metres at GNSS measurements [22]. The elevation difference between the sites in the test network was less than 200 metres.

2.2.3. Signal Obstructions and Multipath. Signal obstructions prevent perfect working GNSS antenna. They could limit the number of visible satellites; also, some of them can increase signal multipath. Metallic objects, lakes, and other reflecting surfaces can cause signal reflection and affect travel time of the signal. For phase measurements and RTK positioning, multipath errors are about 1 to $5 \mathrm{~cm}$. Locating the stations in a clear environment with an open view of the sky could help in reducing multipath. Additionally, a GNSS antenna with a ground plane should be used to help minimize multipath in all sites.

2.2.4. The Geometric Configuration of the Satellites. Satellite geometry is necessary for the correct measurements. The general GNSS design was for unaided code observation and was specific to global coverage with a minimum of 4 satellites above $5^{\circ}$ elevation $99.9 \%$ of the time for all GNSS. In 3-D positioning, phase observations necessitate 4 observable satellites; therefore, short periods are detected in the case that RTK measurements are not possible with one GNSS system. A GNSS combination (GPS/GLONASS) increases the feasibility of the RTK solution. In addition, a weak satellite configuration (satellite distribution in the view of the observer) ends up in an increased dilution of the precision (DOP). Sky blocks such as house walls and tree canopies prevent GNSS observation.

The relations between $\sigma_{\mathrm{r}}$ and the associated standard deviation of positioning $\left(\sigma^{*}\right)$ is described with a scalar quantity (DOP).

$$
\sigma^{*}=\mathrm{DOP} \cdot \sigma_{r}
$$

This criterion has different notations such as horizontal $(\mathrm{H})$, vertical $(\mathrm{V})$, and $3 \mathrm{D}$ positioning $(\mathrm{P}) \mathrm{DOP}$. The horizontal and vertical components are collected under the title of PDOP as the position error induced by satellite geometry. When the PDOP values are between 1 and 2 (confidence level), the positional measurements are considered adequate enough to meet all needs $[10,23]$.
2.2.5. Other Factors. The satellite data for satellite clock offsets and orbits are given in a broadcast message that is taken by the GNSS receivers. The satellite clock offsets have errors $(10 \mathrm{~ns})[23,24]$. However, the effect of satellite clock errors is equal to the rover, which is also the case for the reference station. Finally, the errors are cancelled when network corrections are used. For a single reference station that has a baseline length " $r$," a satellite orbit error " $e_{0}$ " at an $R$ distance to the satellites yields an error in the estimated position, ep, of approximately: ep $=e_{0}{ }^{*} r / R$, which might be derived from the Taylor expansion. For broadcast orbits employed in RTK, it may be accepted that the orbit error is $2 \mathrm{~m}$ ) [23, 24]. If the distance between the reference and the rover is $r=50-100$ $\mathrm{km}$ and $R=20000 \mathrm{~km}$, we have $5-10 \mathrm{~mm}$ errors because of satellite orbits. However, for network RTK with at least 3 reference stations and a linear geographical interpolation, the impact is cancelled to the 1st order and the estimated position (ep) is approximately:

$$
\mathrm{ep} \approx e_{0} *\left(\frac{r}{R}\right)^{2}
$$

For this, the following term is $0.1 \mathrm{~mm}$, and we assume that this error source is equal to zero in the following analysis [23].

\subsection{Test Network and Measurements}

2.3.1. Test Network. A GNSS network was established with six sites for this study. The sites were established in positions that were away from objects that could block the GNSS signals and, therefore, cause signal reflection (building, tree, lake, etc.). The sites were between a distance of 5 and $60 \mathrm{~km}$ according to the AFKU station, which is the LBRTK base station (Figure 1). The NRTK measurements were made by using the CORS-TR network VRS method.

2.3.2. Static Measurements and Analysis. To calculate the coordinates of the sites in the test network, 5 campaign static GNSS measurements were made in 2012-2018. Static survey was employed in the Ashtech Z-Xtreme and Thales Z-Max GPS receivers. The measurements were performed in two days and 8-hour sessions, and the data were recorded with 30 second intervals. One of the most important factors that affect the site position accuracy in RTK measurements is the coordinate accuracy of the base station. Any coordinate error on the base station can affect all sites that calculate coordinates that depend on the base station. Sensitive 


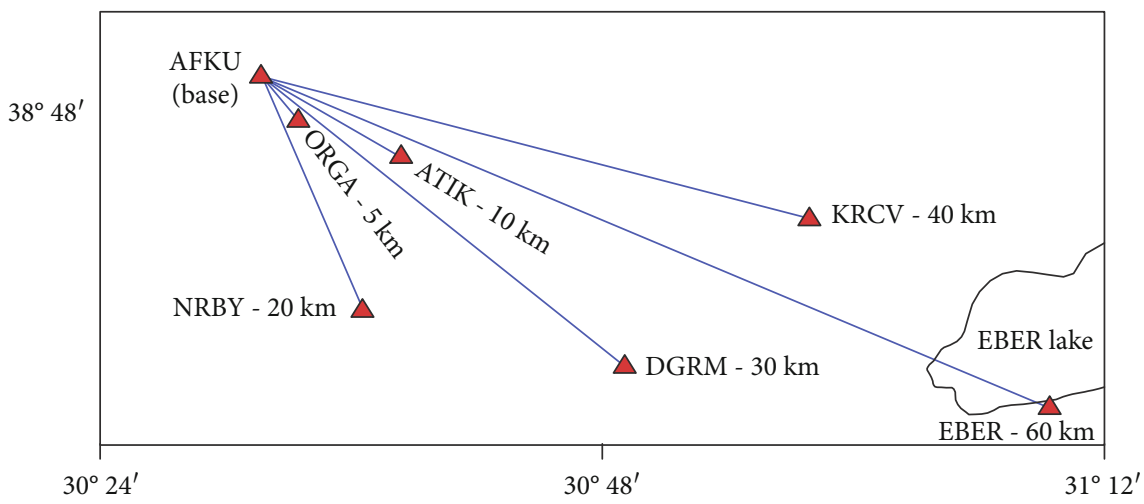

FIGURE 1: The distribution of the stations in the region.

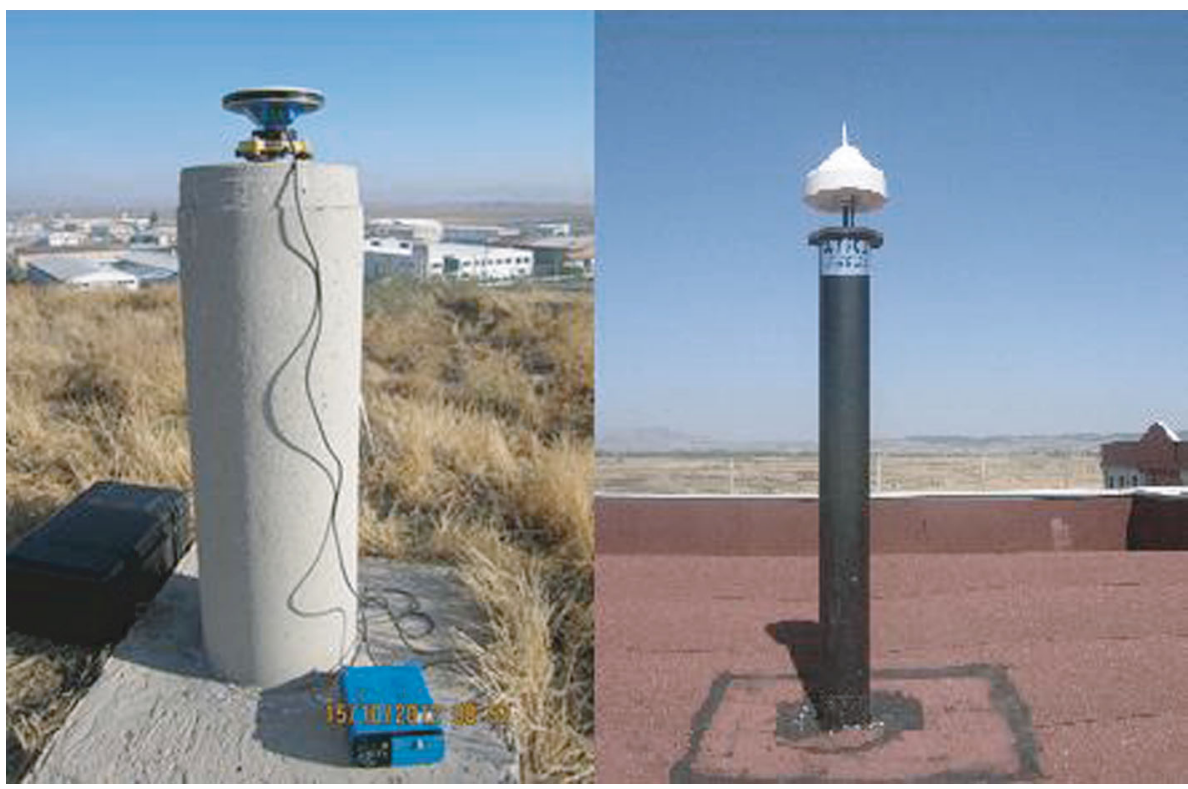

FIgURE 2: Static survey (ORGA-AFKU stations).

coordinates are obtained by examining the temporal change of a site with long-lasting GNSS observations. The sensitive coordinates of the AFKU site, which is the LBRTK base station, were obtained as a result of the evaluation of 3 years of GNSS measurements (Figures 2-4). Blewit and Lavallée [25] recommend that 2.5 years be adopted as a standard minimum data span for velocity solutions intended for tectonic interpretation or reference frame production and that we be sceptical of geophysical interpretations of velocities derived using shorter data spans.

The data were processed by employing GAMIT strategies version 10.5 [26]. The antenna phase centre was derived in line with the height-dependent model. The accurate orbit information (IGS-Final) in SP3 format needed for the GAMIT process was downloaded from the Scripps Orbit and Permanent Array Center (SOPAC) database [26]. The earth rotation parameters (ERPs) came from USNO Bull. B (United States Naval Observatory Bulletin B). In the analyses, LC (L3), the ionosphere-independent linear combination of the L1 and L2 carrier waves, was employed. The FES2004
OTL grid was employed to interpolate the OTL components from a global grid [26-28]. The GAMIT module can estimate 3-D coordinates, satellite orbits, atmospheric zenith delays, and earth rotation parameters using carrier phase measurements and pseudorange observations. GLOBK is used to estimate the velocities in the network. During the estimation of the six-parameter transformation, the generalized constraints were implemented; sixteen IGS network stations used for the stabilization process necessitated the determination of the velocity vectors of the sites $[29,30]$. The Cartesian coordinates and the time series of the sites in the test network were obtained at the end of the process (Table 2, Figure 3).

In Turkey, the ITRF96 datum and 2005.0 reference epochs are used in mapping applications. For this reason, Cartesian coordinate-3-D coordinate conversion was implemented to obtain the ITRF96 datum and moved to the 2005.0 reference epoch.

2.4. Long Base RTK and Network RTK Measurements and Analysis. For long base RTK, the measurements were made 

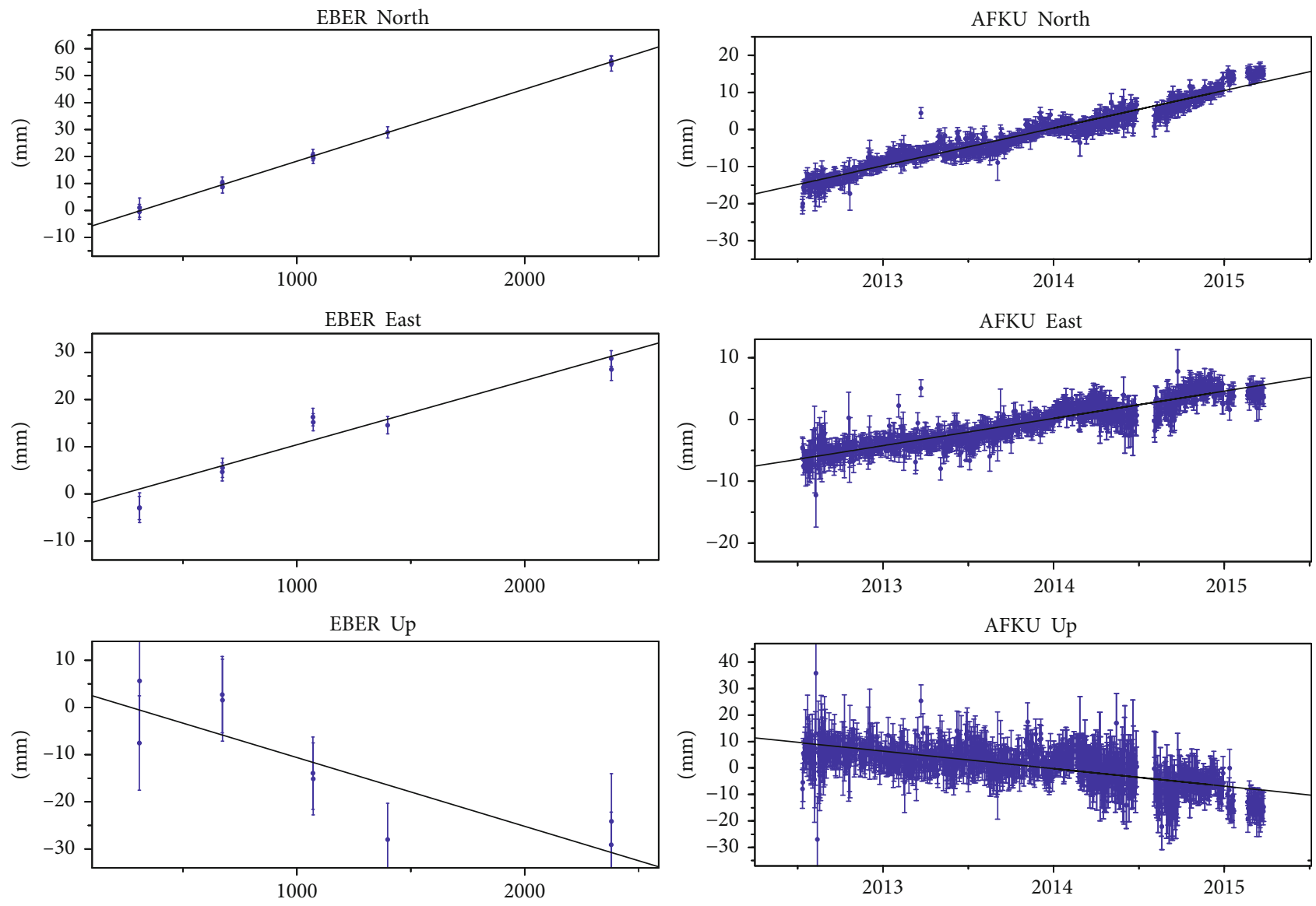

FIgURE 3: EBER and AFKU (LBRTK base station) time series.

relative to the AFKU station that was established by the Department of Geomatics Engineering, Afyon Kocatepe University, in 2012. The Ashtech Proflex 800 GNSS receiver and AERAT1675_120 SPKE geodetic antenna were employed at the AFKU station.

The network RTK measurements were made relative to the CORS-TR Network. CORS-TR has been operating since 2008. The CORS-TR stations were on low-rise and wideplane establishments or on the most appropriate locations in the yards of state-owned plants to ensure 24/7 security and technical assistance [31, 32].

The LBRTK and NRTK measurements were made with STONEX s9. A typical RTK GNSS accuracy may be $8 \mathrm{~mm}+$ $1 \mathrm{ppm}$ horizontally and $15 \mathrm{~mm}+1 \mathrm{ppm}$ vertically [33].

The LBRTK and NRTK measurements were made on $3-4-23-24 / 11 / 2015,20 / 11 / 2017$, and 04/05/2018 days (dd/mm/yyyy) at test network sites based on the CORS-TR and AFKU.

To see the epoch repetition of the LBRTK and NRTK measurements, 300 epoch measurements were collected at each session at 1 second intervals. To be informed on the daily repetitions of the two systems, the measurements from 2015 were made in 4 different days. The $3 \mathrm{rd}$ and 4 th day measurements were made after 20 days to examine the impact of the changes in satellite configurations on the dimensions in 2015. In addition, the measurements of the

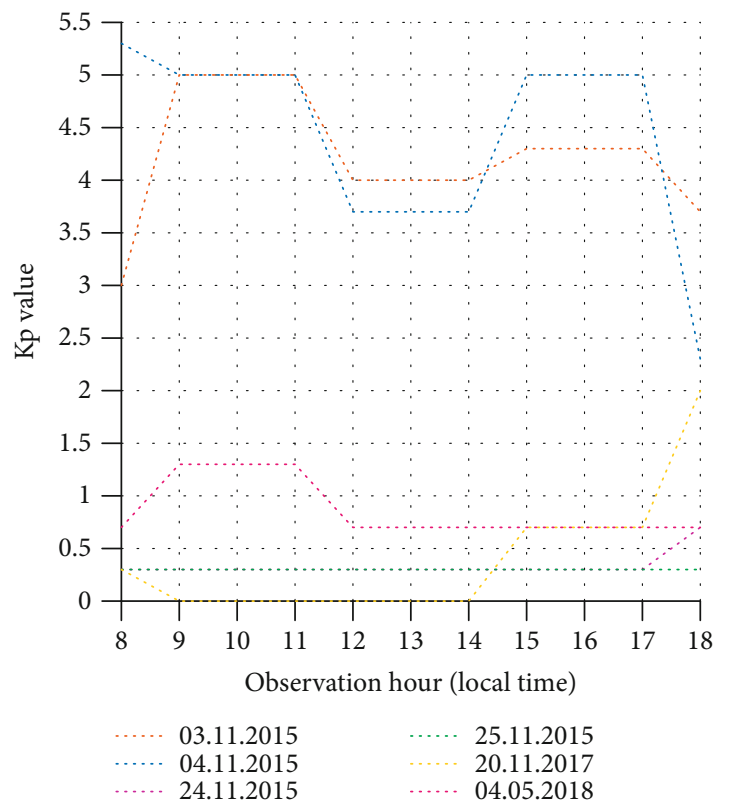

Figure 4: Kp values.

4th day were carried out by postponing them 4 hours to impact of the changes in satellite numbers. On the other hand, to examine the annual repetition of the systems, the 
TABLE 2: Cartesian coordinates and standard deviation of all sites.

\begin{tabular}{lccccccc}
\hline Site & Base length $(\mathrm{km})$ & $X(\mathrm{~m})$ & $Y(\mathrm{~m})$ & $Z(\mathrm{~m})$ & $\begin{array}{c}X \text { sigma } \\
(\mathrm{mm})\end{array}$ & $Y$ sigma $(\mathrm{mm})$ & $Z$ sigma $(\mathrm{mm})$ \\
\hline AFKU & Base & 4286757.450 & 2528392.483 & 3977252.368 & 0.5 & 0.4 & 0.5 \\
ORGA & 3.9 & 4286937.868 & 2531586.207 & 3975043.029 & 2.3 & 1.5 & 2.5 \\
ATIK & 11.1 & 4284857.830 & 2538551.314 & 3973108.734 & 3.6 & 3.0 & 2.0 \\
NRBY & 17.4 & 4291786.359 & 2539642.365 & 3964830.511 & 3.0 & 2.5 & 2.7 \\
DGRM & 31.9 & 4284713.759 & 2556519.698 & 3961520.308 & 2.9 & 2.8 & 2.8 \\
KRCV & 39.2 & 4272677.637 & 2564233.529 & 3969680.650 & 2.8 & 2.6 \\
EBER & 59.1 & 4270979.940 & 2582503.905 & 3959481.466 & 2.3 & 2.5 \\
\hline
\end{tabular}

TABle 3: PDOP values.

\begin{tabular}{|c|c|c|c|c|c|c|}
\hline Site & 03.11.15 PDOP & 04.11.15 PDOP & 24.11.15 PDOP & 25.11.15 PDOP & 20.11.2017 PDOP & 04.05.2018 PDOP \\
\hline$\overline{\text { ORGA }}$ & 1.7 & 1.5 & 1.4 & 1.5 & 2.3 & 2.1 \\
\hline ATIK & 1.6 & 1.5 & 1.7 & 1.7 & 2.3 & 2.4 \\
\hline NRBY & 1.7 & 1.3 & 1.6 & 1.2 & 2.0 & 2.4 \\
\hline DGRM & 1.6 & 1.6 & 1.5 & 1.6 & 2.1 & 2.5 \\
\hline KRCV & 1.8 & 1.9 & 1.5 & 1.5 & 2.0 & 2.2 \\
\hline EBER & 1.4 & 1.4 & 1.6 & 1.7 & 2.3 & 2.5 \\
\hline
\end{tabular}

same measurements were repeated in November 2017 and May 2018. All measurements were carried out after solution of the AR. As mentioned above, one of the factors that affects the RTK measurements is the PDOP values. The PDOP values of all measurements are given in Table 3. In the examinations, it was determined that the PDOP values for measurements made on different days and hours were smaller than 4, and there were no differences among them (Table 3).

The Kp values determined in the measurement days in 2015-2017-2018 are shown in Figure 4. These values were obtained between 08:00 and 18:00 for each site in the test network [34]. It was observed that the $\mathrm{Kp}$ values ranged between 2.3 and 5.3 in the first two measurements made in 2015 (3.11.2015-4.11.2015). It is possible to claim that there is medium magnetism for the days on which the measurements were made. When the other days on which the measurements were made were examined, it was determined that the Kp values were less than 2, indicating stable magnetism for each site in the test network.

To see the epoch repeatability of the LBRTK and NRTK measurements, 300 epoch measurements were collected for each measurement, and the differences in the averages of these measurements were calculated. These differences were visualized with the measurements at (ORGA-5 km) the nearest and (EBER-60 km) the farthest to the AFKU reference station (Figure 5). When the differences in the measurements and the average values were examined, it was determined that there was a max of $1-2 \mathrm{~cm}$ horizontally and a max of $3-4 \mathrm{~cm}$ vertically.

For the daily repetition of the measurements, the first day of measurements for 2015 (3.11.2015) was utilized as the ref- erence in both systems, and the differences between the other days were calculated.

When the daily repetition of the systems was examined, it was determined that there was a change of $\pm 1.5 \mathrm{~cm}$ in the $\mathrm{N}$ and $\mathrm{E}$ measurements. This repetition changed within a max range of $\pm 4 \mathrm{~cm}$ in the "Up" component (Figure 6).

To test the system accuracy, the coordinate differences between LBRTK and NRTK were calculated, and the coordinates were obtained in the static measurements (Figure 7).

\section{Results and Discussion}

In the present study, a test network with 6 sites was established to see the differences between LBRTK and NRTK. The LBRTK and NRTK measurements were made in this test network in 2015 (4 days), 2017, and 2018.

The data of 300 epochs (each) were collected with the LBRTK and NRTK measurements separately at each test site. When considered in general terms, the coordinates obtained from 300 epoch measurements at the sites differed $1-2 \mathrm{~cm}$ horizontally and $3-4 \mathrm{~cm}$ vertically (Figure 5 ). These results indicate that both measurement systems momentarily changed $3-4 \mathrm{~cm}$.

In order to see the daily repetition of LBRTK and NRTK in 2015, the measurements were made in 4 different days. On these measurement days, a medium-level magnetism effect was observed on the first 2 days, and a low-level magnetic effect was observed on day 3 and day 4 (Figure 4). In the LBRTK and NRTK measurements, day 1 was accepted as stable, and the differences between days 2, 3, and 4 were calculated (Table 4 ). When these 

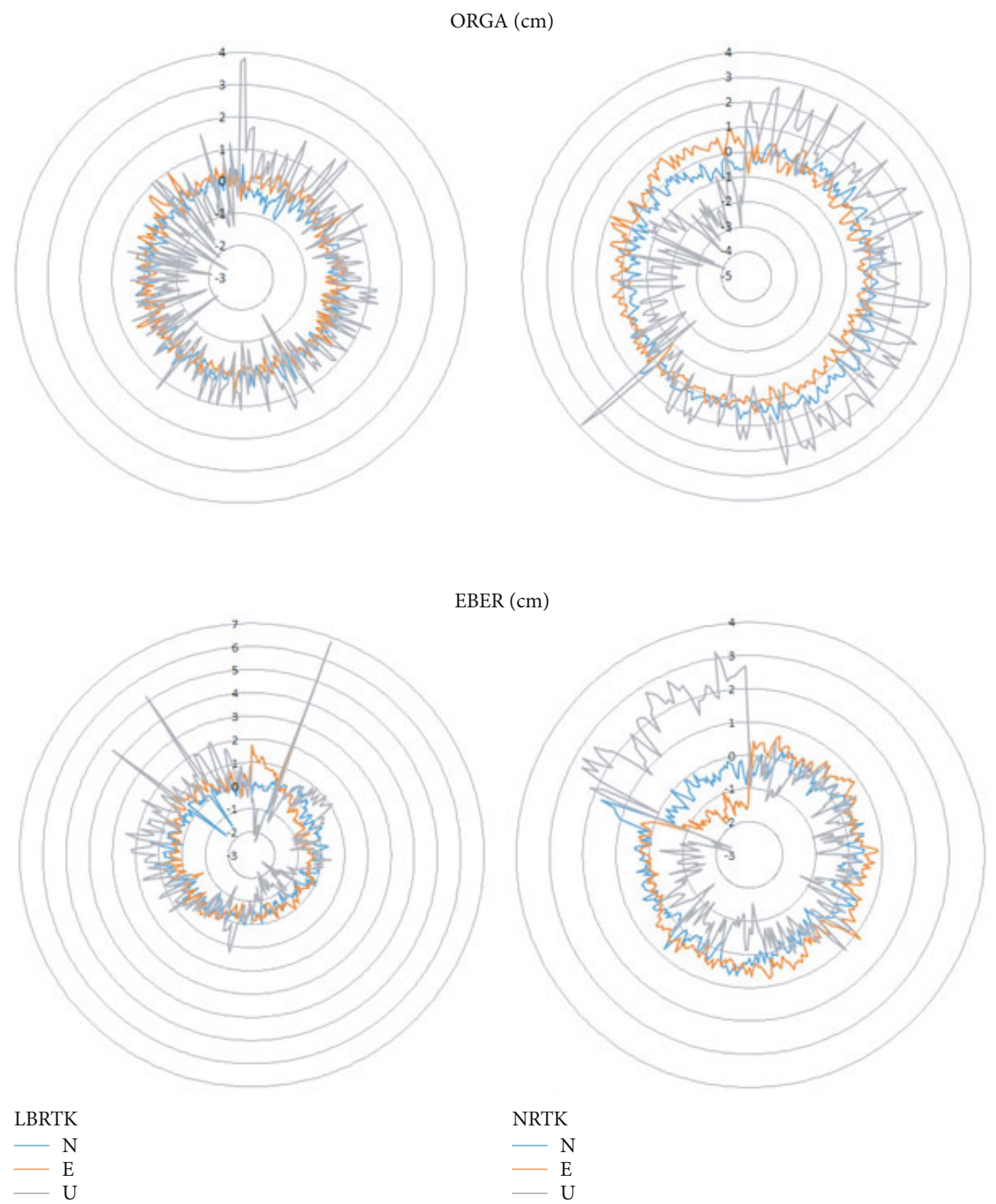

FIgURE 5: Deviations of LBRTK and NRTK coordinates from the average value.

differences were examined, it was estimated that a $\pm 2 \mathrm{~cm}$ horizontal change and $\max$ of $\pm 2 \mathrm{~cm}$ vertical change were determined. Meanwhile, the differences were less than $1 \mathrm{~cm}$ for the East component, and these differences were approximately $\pm 2 \mathrm{~cm}$ for the North component.

In order to see the annual repetition relation between the LBRTK and NRTK systems, the average of the year 2015 was omitted from the measurements in the other years. In addition, to observe the differences between the two systems, the differences in the annual coordinates were taken (Table 4).

When Table 4 is examined, an approximately $2 \mathrm{~cm}$ change is seen in the horizontal repetition of NRTK-LBRTK. In the height component, a difference of approximately $3 \mathrm{~cm}$ is observed in the area up to $50 \mathrm{~km}$. After $50 \mathrm{~km}$, the consistency continued in NRTK, and $7-8 \mathrm{~cm}$ deviations were observed in LBRTK.

When the annual coordinate differences between the two systems were examined, the differences were obtained as $2 \mathrm{~cm}$ horizontally and $3.5 \mathrm{~cm}$ vertically. After $50 \mathrm{~km}$, although no significant changes were observed in the horizontal component, there was a deviation of $5 \mathrm{~cm}$ in the vertical component.

With the static measurements that were made to test the accuracy of the system, the coordinate differences between LBRTK and NRTK were calculated.

As a result of the evaluations made based on Figure 7, it was observed that the LBRTK-static and NRTK-static differences and the differences in the East coordinates at $5 \mathrm{~km}$ distance (ORGA), which is the nearest to the AFKU reference 

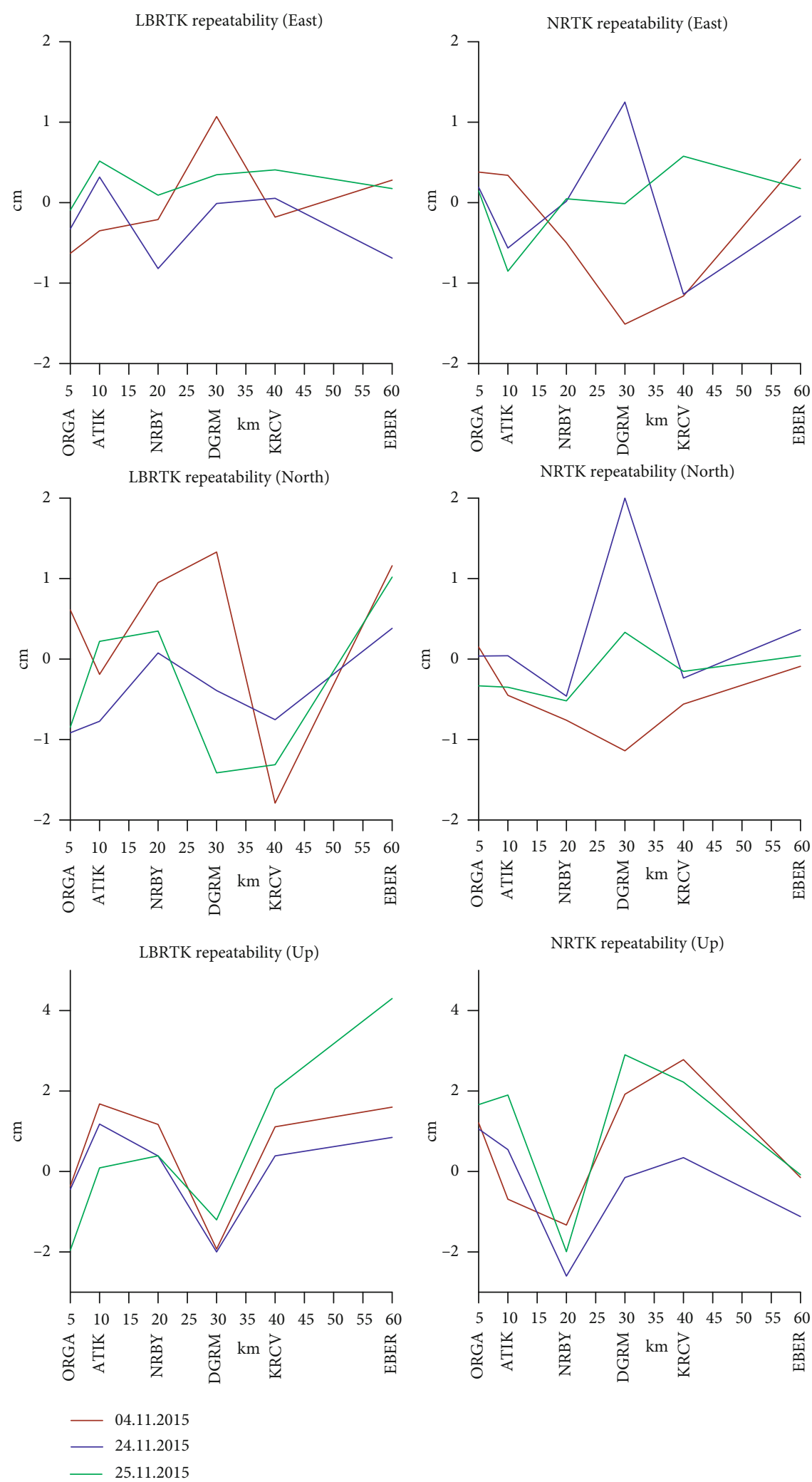

FIGURE 6: Daily repeatability of LBRTK and NRTK.

station, were at a max of $2 \mathrm{~cm}$ in both systems. It was also observed that the differences between the static measurements of both systems at a distance of $10 \mathrm{~km}$ (ATIK) were approximately $2.5 \mathrm{~cm}$. At a distance of $20 \mathrm{~km}$ (NRBY), on the other hand, the differences with respect to the static measurements were a max of $1.5 \mathrm{~cm}$. At the DGRM site, which was located at a distance of $30 \mathrm{~km}$ in the middle of the test network, the differences with the static measurements were close to $2 \mathrm{~cm}$. 

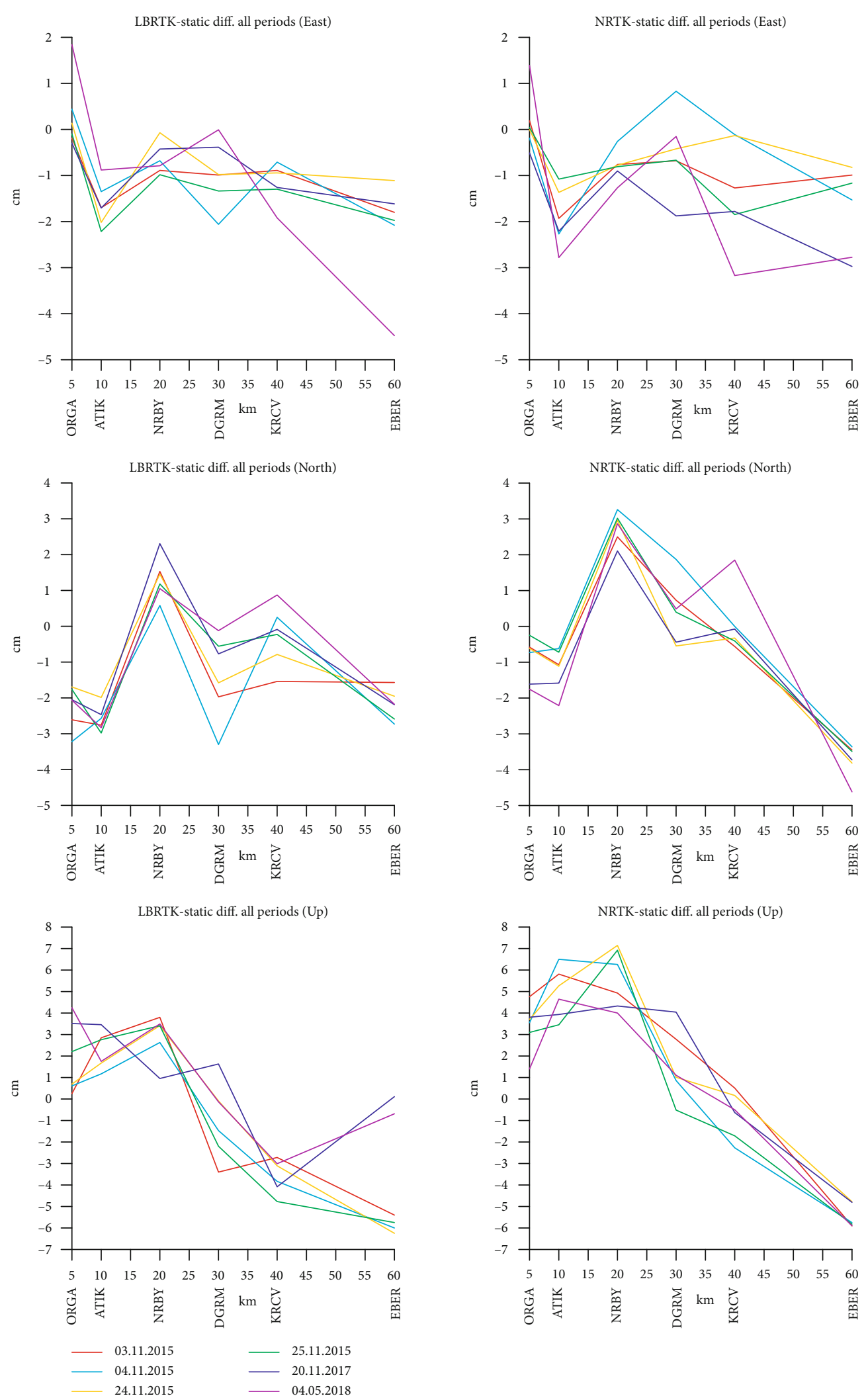

FIGURE 7: Differences between LBRTK, NRTK, and static measurements.

The LBRTK-static difference, which is estimated to increase when moving away from the reference station, was observed to be below $2 \mathrm{~cm}$ at $40 \mathrm{~km}$ (KRCV). The NRTK- static difference progressed to just above $3 \mathrm{~cm}$ at the same site. At the EBER site, which is the latest site of the test network at $60 \mathrm{~km}$, the LBRTK-static difference was observed at 
TABLE 4: Coordinate differences (cm).

\begin{tabular}{|c|c|c|c|c|c|c|c|c|c|c|c|}
\hline & & & & peata & lity ye & & & & & TK-N & TK \\
\hline & & & LBRTK & & & NRTK & & & & & \\
\hline & & $\mathrm{N}$ & $\mathrm{E}$ & $\mathrm{U}$ & $\mathrm{N}$ & $\mathrm{E}$ & $\mathrm{U}$ & & $\mathrm{N}$ & $\mathrm{E}$ & $\mathrm{U}$ \\
\hline & 2015 & 0.0 & 0.0 & 0.0 & 0.0 & 0.0 & 0.0 & & -0.1 & 1.8 & 2.8 \\
\hline ORGA & 2017 & 0.4 & -0.3 & -2.6 & 0.5 & 1.1 & 0.0 & & -0.2 & 0.4 & 0.3 \\
\hline & 2018 & -1.8 & -0.3 & -3.3 & -1.4 & 1.2 & 2.4 & & -0.4 & 0.3 & -2.9 \\
\hline & 2015 & 0.0 & 0.0 & 0.0 & 0.0 & 0.0 & 0.0 & & 0.2 & 1.7 & 3.1 \\
\hline ATIK & 2017 & -0.1 & -0.1 & -1.3 & 0.5 & 0.7 & 1.3 & & -0.5 & 0.9 & 0.5 \\
\hline & 2018 & -0.9 & 0.3 & 0.4 & 1.1 & 1.3 & 0.6 & & -1.9 & 0.6 & 2.9 \\
\hline & 2015 & 0.0 & 0.0 & 0.0 & 0.0 & 0.0 & 0.0 & & 0.0 & 1.7 & 3.3 \\
\hline NRBY & 2017 & -0.2 & -1.1 & 2.4 & 0.2 & 0.8 & 2.3 & Annual coordinate differences between two methods & -0.5 & -0.2 & 3.4 \\
\hline & 2018 & 0.1 & 0.1 & -0.2 & 0.6 & 0.1 & 2.7 & & -0.5 & 1.8 & 0.5 \\
\hline & 2015 & 0.0 & 0.0 & 0.0 & 0.0 & 0.0 & 0.0 & & 1.1 & 2.5 & 1.1 \\
\hline DGRM & 2017 & -1.0 & -1.1 & -1.7 & 1.6 & 1.1 & -3.0 & & -1.5 & 0.3 & 2.4 \\
\hline & 2018 & -1.3 & -1.7 & 0.0 & -0.1 & 0.1 & -0.1 & & -0.1 & 0.6 & 1.2 \\
\hline & 2015 & 0.0 & 0.0 & 0.0 & 0.0 & 0.0 & 0.0 & & 0.1 & 0.3 & 2.3 \\
\hline KRCV & 2017 & 0.3 & -0.5 & 1.0 & 0.9 & -0.2 & -0.2 & & -0.5 & 0.0 & 3.5 \\
\hline & 2018 & 1.0 & -1.4 & -0.1 & 2.3 & -2.2 & -0.3 & & -1.3 & 1.0 & 2.5 \\
\hline & 2015 & 0.0 & 0.0 & 0.0 & 0.0 & 0.0 & 0.0 & & 0.6 & -1.3 & 1.8 \\
\hline EBER & 2017 & -0.1 & 0.0 & -8.5 & 1.8 & 0.2 & -1.7 & & -1.4 & -1.5 & -4.9 \\
\hline & 2018 & 2.7 & 0.0 & -7.7 & 1.6 & 1.1 & -0.6 & & 1.7 & -2.4 & -5.2 \\
\hline
\end{tabular}

$4.5 \mathrm{~cm}$, and the NRTK-Static difference was observed at $3 \mathrm{~cm}$ (Figure 7).

When the differences in the North coordinates were examined, on the other hand, it was observed that although the LBRTK-static difference was a max of $3.5 \mathrm{~cm}$ at ORGA $(5 \mathrm{~km})$, the NRTK-static difference was a max of $2 \mathrm{~cm}$; at the ATIK site $(10 \mathrm{~km})$, these differences were observed to be $3 \mathrm{~cm}$ and $2.5 \mathrm{~cm}$, respectively. At the NRBY site $(20 \mathrm{~km})$, the LBRTK-static measurement difference was a max of $2.5 \mathrm{~cm}$, and the NRTK-static difference was a max of 3.5. At the DGRM site $(30 \mathrm{~km})$, although the LBRTK-static difference was a max of $3.5 \mathrm{~cm}$, the NRTK-static difference was $2 \mathrm{~cm}$.

At the KRCV $(40 \mathrm{~km})$ site, on the other hand, the LBRTKstatic difference was $1.5 \mathrm{~cm}$, and the NRTK-static difference was $2 \mathrm{~cm}$. At the EBER $(60 \mathrm{~km})$ site, which was the latest site, the LBRTK-static difference was observed to be $2.5 \mathrm{~cm}$, and the NRTK-static difference was observed to be $4.5 \mathrm{~cm}$.

When the differences in the Up coordinates were examined in the test network in general, it was observed that the differences for LBRTK-static and NRTK-static were $4 \mathrm{~cm}$ and $5 \mathrm{~cm}$ at the ORGA site, $3.5 \mathrm{~cm}$ and $6.5 \mathrm{~cm}$ at the ATIK site, and $4 \mathrm{~cm}$ and $7 \mathrm{~cm}$ at the NRBY site, respectively.

At the midpoint of the test network (DGRM), the differences were observed at $3.5 \mathrm{~cm}$ and $4 \mathrm{~cm}$; at the KRCV site, the differences were $5 \mathrm{~cm}$ and $2.5 \mathrm{~cm}$. At the farthest site of the test network (EBER), it was observed that the difference was $6 \mathrm{~cm}$.

\section{Conclusions}

In the present study, different from the other studies in the literature, the purpose was to examine and compare the
LBRTK and NRTK methods in all relevant aspects. The epoch repetition, daily repetition, annual repetition, ionospheric effects, and satellite geometry factors, which might affect the LBRTK and NRTK methods, were examined in detail and presented in graphics. The velocities of all stations were calculated, and the sites were converted into the same datum. In the literature, it has been stated that the effect of the height difference is limited in GNSS measurements below 300 metres [22]. Because the elevation difference between the sites in the test network was less than 200 metres and because the area had a flat and smooth topography, the altitude impact was ignored. However, it was also observed that there were no abnormal changes in the LBRTK and NRTK measurements that had different ionospheric values in the midlatitude zone.

When the results were examined, it was observed that the LBRTK and NRTK methods yielded similar results at base lengths up to $40 \mathrm{~km}$. These results were limited to $3 \mathrm{~cm}$ vertically and $2 \mathrm{~cm}$ horizontally. In bases that were longer than $40 \mathrm{~km}$, the deviations started between the LBRTK and NRTK methods, which are accepted as the reference (Figures 6 and 7 ). These results supported the results of other studies. These deviations were observed at $3 \mathrm{~cm}$ horizontally and $5 \mathrm{~cm}$ vertically on a 60-kilometre basis. It was estimated that these deviations will move in proportion to the base length on longer bases. It was stated that the accuracy of the CORS-TR system with which the NRTK measurements were made was nearly $3 \mathrm{~cm}$ in the horizontal direction and $5 \mathrm{~cm}$ in the vertical direction [35]. When this situation was considered, making a measurement with LBRTK at a radius of $40 \mathrm{~km}$ with a stable-working reference station as the centre will suffice for many applications in which 3-D position data are required. 
It was determined that the LBRTK method was more economical on bases that have an average of up to 40 kilometres when the establishment, monitoring, maintenance, and repair costs of the sites required for the NRTK method are considered. Approximately 100 LBRTK systems are established in Turkey. If these systems are regularly monitored, it can be used as an effective surveying tool in $3 \mathrm{D}$ positioning and mapping [36].

\section{Data Availability}

The data required to reproduce these findings cannot be shared at this time as the data also forms part of an ongoing study.

\section{Conflicts of Interest}

The authors declare no conflict of interest regarding the publication of this paper.

\section{Acknowledgments}

This study was supported by Afyon Kocatepe University Scientific Research Projects Coordination Department (Project No: 15.MUH.01). The authors are grateful to the students of Geomatics Engineering of Afyon Kocatepe University for their support of the GNSS measurements. The authors are grateful to MIT for providing the license of the GAMIT/GLOBK software.

\section{References}

[1] M. Berber, A. Ustun, and M. Yetkin, "Comparison of accuracy of GPS techniques,” Measurement, vol. 45, no. 7, pp. 17421746, 2012.

[2] M. Berber and N. Arslan, "Network RTK: a case study in Florida," Measurement, vol. 46, no. 8, pp. 2798-2806, 2013.

[3] P. J. G. Teunissen, D. Odijk, and B. Zhang, "PPP-RTK: results of CORS network-based PPP with integer ambiguity resolution," Journal of Aeronautics, Astronautics and Aviation, Series A, vol. 42, no. 4, pp. 223-230, 2010.

[4] B. Zhang, P. J. G. Teunissen, and D. Odijk, "A novel undifferenced PPP-RTK concept," Journal of Navigation, vol. 64, no. S1, pp. S180-S191, 2011.

[5] B. Zhang, Y. Chen, and Y. Yuan, "PPP-RTK based on undifferenced and uncombined observations: theoretical and practical aspects," Journal of Geodesy, vol. 93, no. 7, pp. 1011-1024, 2019.

[6] G. Hu, D. A. Abbey, N. Castleden et al., "An approach for instantaneous ambiguity resolution for medium-to longrange multiple reference station networks," GPS Solutions, vol. 9, no. 1, pp. 1-11, 2005.

[7] D. Kim and R. B. Langley, "Long-range single-baseline RTK for complementing network-based RTK," in ION GNSS 20th International Technical Meeting of the Satellite Division, pp. 639-650, Fort Worth, TX, USA, 2007.

[8] S. Ögütçü and İ. Kalaycı, "Accuracy and precision of networkbased RTK techniques as a function of baseline distance and occupation time," Arabian Journal of Geosciences, vol. 11, no. $13,2018$.
[9] C. Rizos and S. Han, "Reference station network based RTK systems-concepts and progress," Wuhan University Journal of Natural Sciences, vol. 8, no. 2, pp. 566-574, 2003.

[10] U. Vollath, H. Landau, X. Chen, K. Doucet, and C. Pagels, "Network RTK versus single base RTK-understanding the error characteristics," in Proceedings of the 15th International Technical Meeting of the Satellite Division of the Institute of Navigation, pp. 24-27, Portland, Oregon, USA, September 2002.

[11] P. Wielgosz, I. Kashani, and D. Grejner-Brzezinska, “Analysis of long-range network RTK during a severe ionospheric storm," Journal of Geodesy, vol. 79, no. 9, pp. 524-531, 2005.

[12] L. Wanninger, "The performance of virtual reference stations in active geodetic GPS-networks under solar maximum conditions," Proceedings of the 12th International Technical Meeting of the Satellite Division of the Institute of Navigation, 1999, pp. 1419-1427, Nashville, TN, USA, 1999.

[13] K. Gumus, M. O. Selbesoglu, and C. T. Celik, "Accuracy investigation of height obtained from classical and network RTK with ANOVA test," Measurement, vol. 90, no. 8, pp. 135$143,2016$.

[14] S. Ögütçü, "Temporal correlation length of network based RTK techniques," Measurement, vol. 134, no. 2, pp. 539-547, 2019.

[15] A. El-Mowafy, "Precise real-time positioning using network RTK," in Global Navigation Satellite Systems - Signal, Theory and Applications, pp. 161-188, Shanghai Astronomical Observatory, Wuhan, China, 2012.

[16] N. O. Aykut, E. Gülal, and B. Akpinar, "Performance of single base RTK GNSS method versus network RTK," Earth Sciences Research Journal, vol. 19, no. 2, pp. 135-139, 2015.

[17] B. Park and C. Kee, “The compact network RTK method: an effective solution to reduce GNSS temporal and spatial decorrelation error," Journal of Navigation, vol. 63, no. 2, pp. 343$362,2010$.

[18] D. Twaddle, Traditional GNSS RTK Compared to Network RTK Solutions, 2018, https://www.ntspatial.com/uploads/6/6/ 3/1/6631203/dtwaddle_base_v_cors_comparison_25.27.pdf.

[19] K. Guo, Y. Liu, Y. Zhao, and J. Wang, "Analysis of ionospheric scintillation Characteristics in Sub-Antarctica Region with GNSS Data at Macquarie Island," Sensors, vol. 17, no. 12, p. 137, 2017.

[20] L. Wanninger, "Effects of the equatorial ionosphere on GPS," GPS World, vol. 4, no. 7, pp. 48-54, 1993.

[21] M. Rupprecht, Orbit propagation, 2016, http://www.dk3wn .info/propagation.shtml.

[22] K. Hastaoğlu and D. U. Şanlı, "The factors affecting the precision of GPS vertical positioning: inference regarding the studies of GPS leveling and local GPS geoid (In Turkish)," in 2nd. National Engineering Measurements Symposium, pp. 466-476, İstanbul, Turkey, 2005.

[23] T. Emardson, P. O. J. Jarlemark, S. Bergstrand, T. Nilsson, and J. Johansson, Measurement Accuracy in Network-RTK, SP Technical Research Institute of Sweden, Göteborg, Sweden, 2009.

[24] D. Sathyamoorthy, S. Shafii, Z. F. M. Amin, A. Jusoh, and S. Z. Ali, "Evaluating the Effect of Global Positioning System (GPS) Satellite Clock Error via GPS Simulation," in IOP Conference Series: Earth and Environmental Science, vol. 37, June 2016no. 1. 
[25] G. Blewitt and D. Lavallée, "Effect of annual signals on geodetic velocity," Journal of Geophysical Research, vol. 107, no. B7, pp. ETG 9-1-ETG 9-11, 2002.

[26] T. A. Herring, R. W. King, and S. C. McClusky, "GAMIT Reference Manual, GPS Analysis at MIT, Release 10.4," in Department of Earth, Atmospheric and Planetary Sciences, Massachusetts Institute of Technology, Cambridge, USA, 2010.

[27] H. Ozener, A. Dogru, and A. Unlutepe, “An approach for rapid assessment of seismic hazards in Turkey by continuous GPS data," Sensors, vol. 9, no. 1, pp. 602-615, 2009.

[28] F. Lyard, F. Lefevre, T. Letellier, and O. Francis, "Modelling the global ocean tides: modern insights from FES2004," Ocean Dynamics, vol. 56, no. 5-6, pp. 394-415, 2006.

[29] E. Gülal, H. Erdoğan, and İ. Tiryakioğlu, "Research on the stability analysis of GNSS reference stations network by time series analysis," Digital Signal Processing, vol. 23, no. 6, pp. 1945-1957, 2013.

[30] I. Tiryakioğlu, "Geodetic aspects of the 19 May 2011 Simav earthquake in Turkey," Geomatics, Natural Hazards and Risk, vol. 6, no. 1, pp. 76-89, 2013.

[31] K. Eren, T. Uzel, E. Gülal, O. Yıldırım, and A. Cingöz, "Results from a comprehensive global navigation satellite system test in the CORS-TR network: case study," Journal of Surveying Engineering, vol. 135, no. 1, pp. 10-18, 2009.

[32] T. Uzel, K. Eren, E. Gulal, I. Tiryakioglu, A. A. Dindar, and H. Yilmaz, "Monitoring the tectonic plate movements in Turkey based on the national continuous GNSS network," Arabian Journal of Geosciences, vol. 6, no. 9, pp. 3573-3580, 2013.

[33] D. Electronics, Stonex User Manual, 2017, http://www .dogaelektronik.com.tr/uploads/products/support/brochures/ s900t-gnss/s900-brochure-eng.pdf.

[34] U. Sezen, F. Arikan, O. Arikan, O. Ugurlu, and A. Sadeghimorad, "Online, automatic, near-real time estimation of GPS-TEC: IONOLAB-TEC," Space Weather, vol. 11, no. 5, pp. 297-305, 2013.

[35] S. Bakıc1, Reference GNSS Stations in Turkey (In Turkish), 2018, http://tujk2017.boun.edu.tr/sites/default/files/bildiriler/SBAKICI_TUJK2017.pdf.

[36] I. Tiryakioğlu, A. A. Dindar, B. Akpınar et al., Processing Data of Continuously GNSS Stations and Publishing in WEB Environment: Generating Data Processing Center (In Turkish), 2017, http://tujk2017.boun.edu.tr/sites/default/files/bildiriler/ibrahim_tiryakioglu_PEGASUS.pdf. 


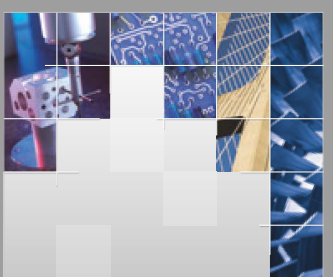

\section{Enfincering}
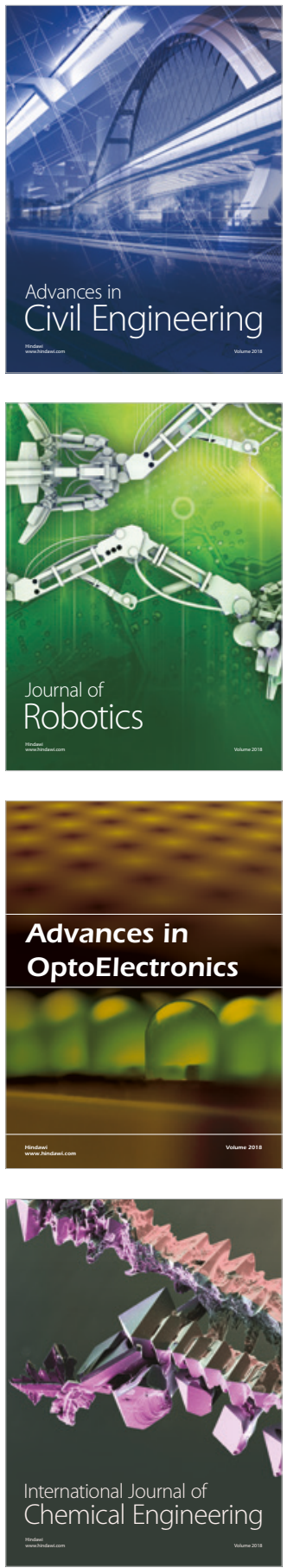

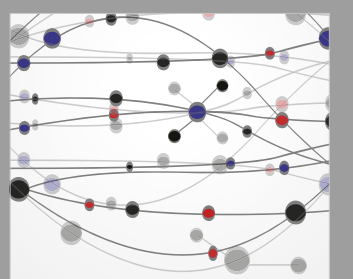

\section{Rotating \\ Machinery}

The Scientific World Journal

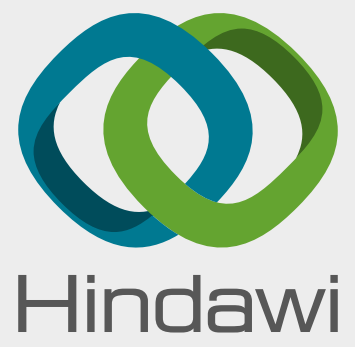

Submit your manuscripts at

www.hindawi.com
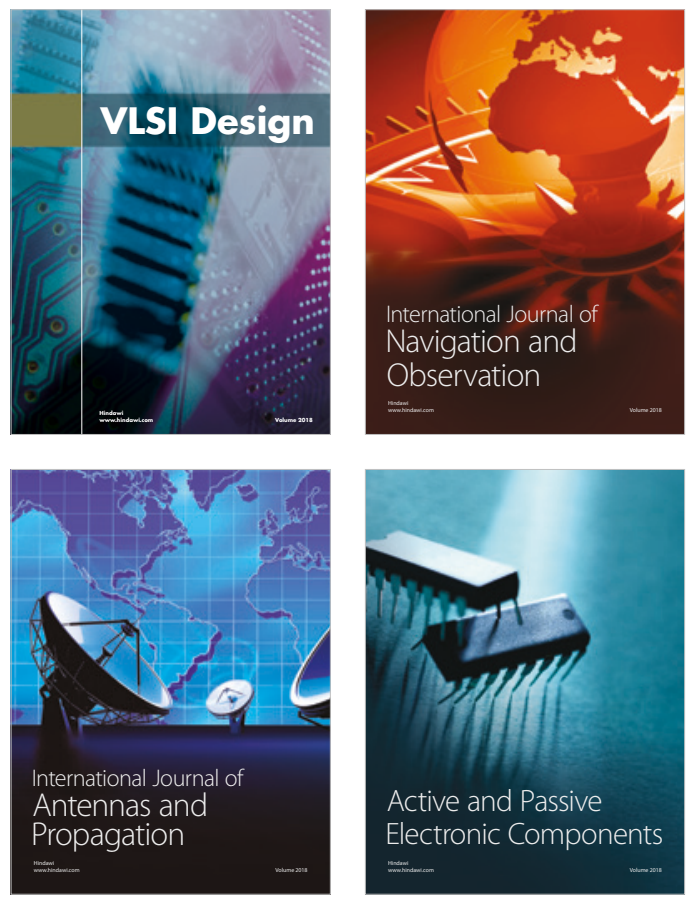
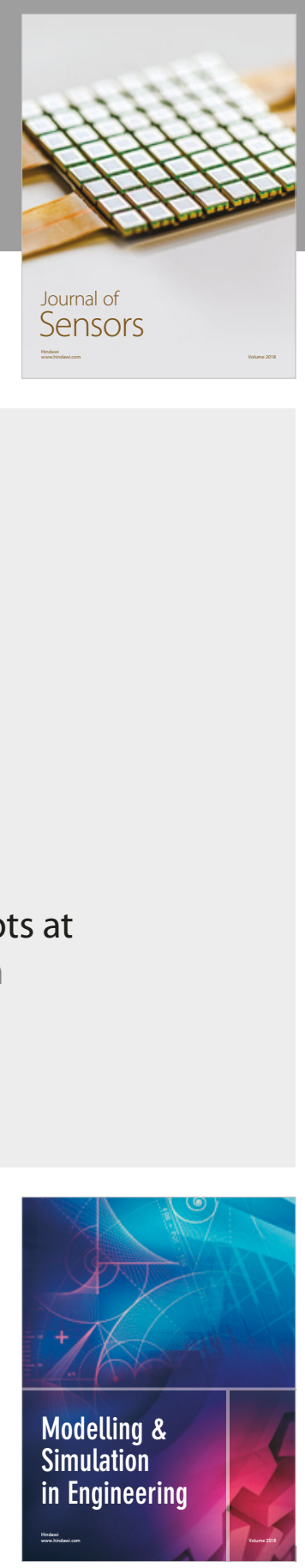

\section{Advances \\ Multimedia}
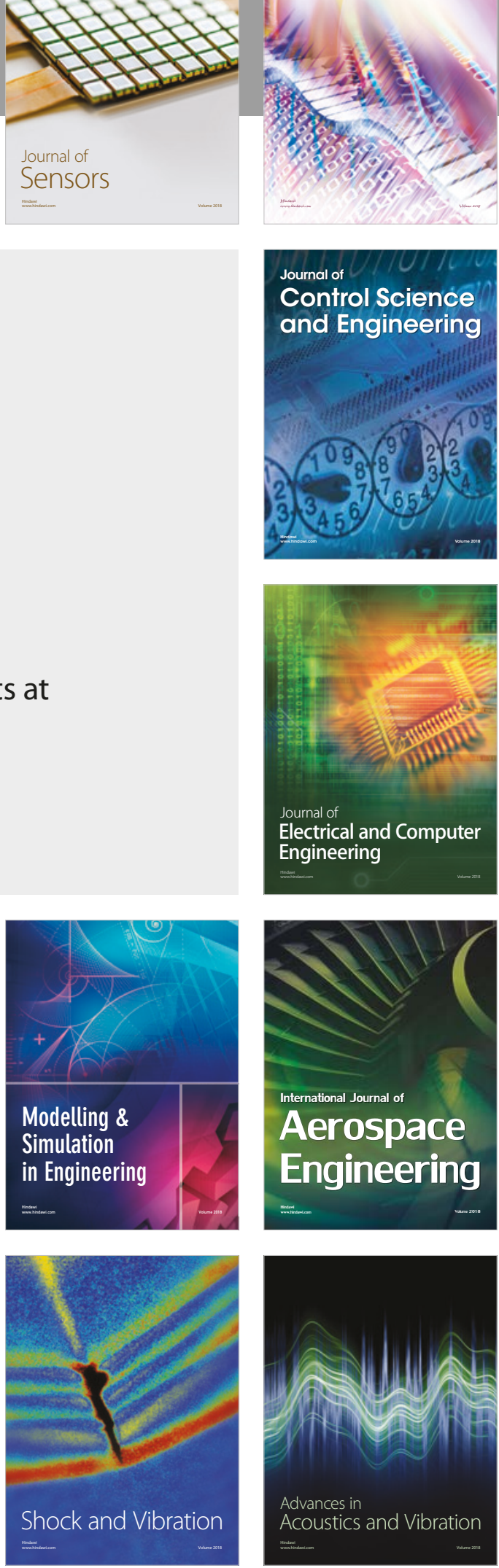